\title{
PERSPECTIVAS LABORALES DE LA MUJER EN ESPAÑA
}

\author{
José Ignacio Casas y M. Ángeles Sallé
}

Al examinar la evolución reciente del mercado de trabajo en España, uno de los fenómenos reseñados con más frecuencia es el incremento brusco de la población activa femenina, tanto en términos relativos como absolutos, durante los dos últimos años. Según las cifras disponibles sobre el volumen de mano de obra, se habría producido una inexplicable explosión en la participación laboral de la mujer, de modo que entre el cuarto trimestre de 1985 y el mismo período de 1987 la población activa femenina creció un 17,0 por ciento, mientras que la masculina lo hizo sólo en un 1,3 por ciento. A su vez, estos incrementos han repercutido de modo muy diferente en lo que respecta a la composición de la población ocupada y desempleada. Durante el bienio señalado, el número de mujeres con una ocupación ha crecido un 13,1 por ceinto, mientras que el de varones lo ha hecho en un 6,3 por ciento. En cambio, la población desempleada femenina creció fuertemente (un 28,2 por ciento), cuando la masculina disminuyó en un 18,9 por ciento. ${ }^{1}$

En cualquier caso, la tasa de actividad femenina seguía siendo a finales de 1987 de un 32,1 por ciento (frente a un 67,6 por ciento de los varones), con una tasa de desempleo del 27,9 por ciento $(16,0$ por ciento de los

1. Los cálculos se basan en la Encuesta de Población Activa, según la revisión de las series realizada por el propio INE a partir de la nueva metodología utilizada desde el segundo trimestre de 1987. 
varones), lo que arroja una tasa de empleo ${ }^{2}$ para la mujer del 23,2 por ciento (56,7 por ciento para los varones).

Estas divergencias tan acentuadas en la evolución de uno y otro colectivo muestran, una vez más, que el análisis de la participación laboral de la mujer (y a la postre también la del varón) no puede abordarse aplicando acríticamente el modelo clásico - masculino- de comportamiento laboral.

Los elementos que concurren en la configuración de la mano de obra masculina, su composición interna, las pautas laborales a lo largo del ciclo de vida, etc., son muy diferentes a los que pueden encontrarse en el caso de la mano de obra femenina. Estas diferencias no pueden atribuirse a un alejamiento pretendidamente voluntario del mundo del trabajo por parte de las mujeres ya que, se argumenta así, sus prioridades estarían de otro lado. Pero no es éste el lugar para revisar las diversas teorías sobre la cuestión, ${ }^{3}$ sino de señalar los factores que, en el caso español, provocan la resultante antes apuntada.

Porque, en efecto, la actividad laboral de la mujer es muy dilatada e intensa, tanto en la jornada diaria como a lo largo de todo el transcurso de la vida. Pero el tipo de actividad es sustancialmente distinto del prototipo de la actividad laboral masculina, aunque las razones de esta divergencia son estructurales y atañen tanto al varón como a la mujer.

\section{ECONOMIA Y FAMILIA}

Por lo que se refiere al caso de España, el primer elemento a tener en cuenta es el tipo de estructura familiar. El modelo de familia presenta una estructura fuertemente jerarquizada, con una notable subordinación y dependencia del cónyuge (mujer) respecto al cabeza de familia (varón) y, a su vez, de los hijos respecto a los padres. En este esquema han influido, por un lado, los largos años de ideología conservadora, llevada a su extremo durante el período franquista; y, por otro lado, el escaso y tardío desarrollo de servicios sociales y asistenciales por parte del Estado. Ello ha llevado a que la unidad familiar siga siendo en buena parte el lugar en el que se produce y administra un volumen importante de

2. Porcentaje de ocupados respecto de la población total. Las cifras son de EPA, cuarto trimestre de 1987, principales resultados.

3. Un análisis de los enfoques teóricos básicos puede verse en Casas (1988). 
bienes y servicios, que en otras economías formarían parte del sector público. ${ }^{4}$

Junto a la importancia relativa del trabajo doméstico, hay que destacar también el papel de la legislación laboral franquista, claramente discriminatoria respecto a la mujer. Por ejemplo, la dote, compensación económica que la empresa daba a las mujeres que abandonaban su trabajo al casarse, no fue derogada hasta la promulgación de la Constitución en 1978, y hasta poco antes (1975) las casadas debían contar con el consentimiento del marido para poder ejercer un empleo.

$\mathrm{Si}$, además, tenemos en cuenta la tradicional incapacidad de la economía española para generar un volumen suficiente de puestos de trabajo, nos encontramos con un esquema de relación entre familia y economía caracterizado por:

1. Una importancia desmesurada de la unidad familiar como productora de bienes y servicios, bien destinados al consumo de los miembros de la familia (trabajo doméstico), bien destinados al mercado fuera del hogar. Este último aspecto explica el alto porcentaje de «ayuda familiar» dentro de la población ocupada, de modo que a finales de 1987 el 6,8 por ciento de la misma se encuentra en esa situación, porcentaje que se eleva al 13,2 por ciento para la población ocupada femenina. ${ }^{5}$

2. Unas fuertes barreras de acceso al mercado de trabajo, sobre todo al mercado de trabajo asalariado, de forma que a pesar de la débil generación de puestos de trabajo, el precio de la mano de obra (salarios) y la renta familiar correspondiente se han mantenido a niveles superiores a los que cabría esperar. Así, la relación de dependencia (número de personas dependientes de cada persona que trabaja) es particularmente alta y se ha elevado a partir de la crisis económica (Alcaide, 1981).

3. La consecuencia de lo anterior sobre las relaciones en el seno de la familia es doble. Por un lado se refuerza la jerarquización de roles en la estructura familiar, de modo que el cabeza de familia-varón ve realzado su papel de bread-winner (el que gana el sustento para la familia) a expensas de los demás miembros. Éstos, en particular la mujer, deberán de-

4. En Gershuny (1983) se encuentra un análisis detallado de estos aspectos. Para España puede consultarse Durán (1988).

5. EPA, cuarto trimestre de 1987 , principales resultados. A estas cifras habría que añadir casi otro tanto número de mujeres que estadísticamente figuran como empresarias sin asalariados en la agricultura y el comercio, pero que en realidad trabajar en el negocio o explotación familiar de forma dependiente. 
dicar un esfuerzo mayor a mejorar el valor de mercado de aquél, con el fin de que el salario que perciba compense la baja participación laboral de los restantes miembros. Este dualismo y dependencia en el seno de la familia ha hecho posible una disponibilidad laboral acrecentada del cabeza de familia a través de, por ejemplo, los bien conocidos fenómenos de las migraciones internas y externas, el recurso a las horas extraordinarias, etc., durante los años sesenta y mediados de los setenta. ${ }^{6}$

\section{TENDENCIAS EN EL MERCADO DE TRABAJO FEMENINO}

El mercado laboral español aparentemente no había sufrido transformaciones estructurales importantes hasta la reforma que se puso en marcha a partir de los años ochenta. Sin embargo, durante las últimas décadas se han ido produciendo modificaciones drásticas en la composición de la mano de obra, en particular la femenina, por debajo de unas cifras globales que mostraban una gran estabilidad.

Desde los años sesenta han surgido nuevos elementos que, sin modificar el esquema familiar señalado en el apartado anterior, han ido mostrando las tensiones existentes en éste. En primer lugar, el declive de la agricultura tradicional ha expulsado progresivamente mano de obra subempleada, en especial mano de obra femenina, en el seno de una población de edad relativamente avanzada y bajo nivel educativo. Estas características suponen, por sí mismas, una disminución de la mujer en la población activa, fenómeno por lo demás típico de economías en desarrollo.

De igual importancia en lo que respecta a la composición de la mano de obra femenina ha sido el boom educativo de los años setenta, que ha transformado la oferta de trabajo femenino. ${ }^{7}$ Las oportunidades de nuevos empleos durante este período han sido en general escasas, a excepción del crecimiento (inferior que en otros países desarrollados) de los servicios de bienestar público. Esta debilidad de la demanda de mano de obra ha provocado tres fenómenos complementarios: ${ }^{8}$

a) Una segregación ocupacional elevada, de modo que, por un lado, la presencia femenina en la industria —incluso en las ramas «femeninas»

6. El análisis de la dinámica salarial durante esos años puede verse en Serrano y Malo de Molina (1979), y Malo de Molina (1983).

7. Para un análisis de este punto, véase Casas (1987:42-52).

8. La descripción de la situación que sirve de base a lo que sigue puede verse en Casas (1987). 
del textil, confección y calzado- se va reduciendo; y, por otro lado, se produce una hiperconcentración en el sector servicios (enseñanza, sanidad y servicios personales).

b) Una fuerte selectividad, es decir, discriminación en el acceso de la mujer al mercado de trabajo. Esta circunstancia ha producido como resultante que sólo un perfil muy definido de las demandantes reales y potenciales de trabajo remunerado accedan a un empleo. Es decir, el tipo de mujer ocupada está mucho más alejado de los rasgos socio-demográficos generales de la población femenina que lo que pueda ocurrir en el caso de la población masculina. De este modo, la segmentación y disparidades internas de la fuerza de trabajo femenina, incluso desde el punto de vista de la distribución territorial, son notablemente más agudas que entre los varones.

c) Un crecimiento inusitado del desempleo femenino (junto al paro desanimado y al subempleo). Esta circunstancia ha venido reforzada por el hecho de que en España el paro es fundamentalmente juvenil; es decir, se produce en los tramos de edad donde se concentra la mayor y más cualificada oferta de mano de obra femenina. La crisis económica ha producido así un drástico frenazo al proceso de incorporación de la mujer al mercado de trabajo.?

Desde el punto de vista de la evolución de las estructuras. familiares, las repercusiones de la tendencia al aumento de la presencia femenina en el mercado de trabajo son en cierta medida contradictorias. En efecto, a pesar del incremento en el número de mujeres que trabajan fuera del hogar, no se ha producido en lo sustancial un nuevo reparto de las tareas domésticas entre los componentes de la familia. La tensión derivada de los requerimientos de la doble jornada laboral de la mujer (dentro y fuera del hogar), se ha resuelto en buena parte a través de un descenso vertical de las tasas de nupcialidad i fecundidad. ${ }^{10}$ Es decir, el modelo familiar no se transforma, sino que su puesta en práctica se retrasa, pero la distribución de roles se mantiene tal como venía produciéndose. Los efectos específicos varían en función de los niveles sociales implicados. Así, por ejemplo, en los estratos sociales profesionales e intermedios, el recurso al servicio doméstico se sigue manteniendo, aunque evolucione su modalidad concreta (asistentas por horas, etc.).

9. Véase sobre este punto a De Miguel (1988).

10. Hasta el año 1978, véase el análisis de Agüiero y Olano (1982). Desde entonces la tendencia ha continuado en el mismo sentido. 
Pero en general el desempleo, marcadamente juvenil y femenino, ha reforzado el esquema tradicional de una sola persona ocupada (el cabeza de familia-varón) por núcleo familiar. De todas las viviendas familiares con más de un habitante y en las que alguno de ellos tiene una ocupación, en el 61,7 por ciento de los casos hay sólo una persona ocupada, frente al 38,3 por ciento en que hay dos o más en esa situación. ${ }^{11}$

\section{ALGUNAS TRANSFORMACIONES RECIENTES}

\section{Politicas de reducción de la oferta de trabajo}

La incidencia de las políticas de intervención y reforma del mercado de trabajo, puestas en marcha durante los últimos años por la mayoría de los países del área de la OCDE, no es fácil de evaluar en lo que respecta a la situación laboral de la mujer.

Por un lado, las políticas que tratan de disminuir los niveles de desempleo, mediante la prolongación del período de escolarización y el adelantamiento de la edad de la jubilación, favorecen el aumento de la relación de dependencia anteriormente señalada. Se estrecha así el ciclo de vida laboral, lo que implica una dedicación mucho más intensiva en un espacio más corto de tiempo. Esta dinámica presiona hacia una distribución de los toles en la familia y la pareja, de modo que ambos cónyuges inviertan en la cartera profesional del cabeza de familia-varón en detrimento de una posible distribución más equilibrada de esfuerzos laborales. ${ }^{12}$ El análisis de las tasas de actividad por grupos de edad (Casas, 1987:1329) muestra cómo el ciclo laboral preparación/dedicación/jubilación, con una separación nítida entre cada una de las tres fases, se ajusta sobre todo a las pautas laborales de los varones, pero no tanto de las mujeres. En el caso de éstas, la actividad de trabajo (dentro y fuera del mercado laboral) se distribuye más homogéneamente a lo largo de todas las etapas de la vida.

11. EPA, segundo trimestre de 1987 , resultados detallados.

12. Estudios sobre este tipo de dinámica pueden verse en Pahl y Pahl (1971) y Finch (1983). 


\section{Políticas de fomento de la contratacion}

En lo que se refiere a las políticas positivas de fomento del empleo, los programas de contratación incentivada o con especiales facilidades para la misma han experimentado en España un impulso poderoso a partir de 1985. La evaluación de su importancia real para el empleo femenino (y masculino) es dif́́cil de realizar, debido a la ausencia de datos fundamentales referidos a los mecanismos de reclutamiento de las personas incluidas en cada uno de los programas de fomento del empleo, la duración de los contratos según el sexo de la persona contratada, los efectos sobre la situación laboral ex post, etc. De esta forma se desconoce absolutamente la eficacia auténtica de los programas de fomento del empleo. En efecto, la finalidad declarada de muchos de estos programas era suministrar una primera experiencia laboral que mejorara objetivamente la capacida de algunos de los llamados «grupos desfavorecidos en el mercado de trabajo» para encontrar un empleo.

El único dato disponible desagregado por sexo es el número de personas contratadas a través de cada uno de los programas. Para 1987, el 30,3 por ciento de los contratos realizados por esta vía se realizaban a mujeres. ${ }^{13}$ Si se tiene en cuenta que en ese mismo año sólo el 26,8 por ciento de la totalidad de contratos en cualquiera de sus modalidades afectó al colectivo femenino, puede destacarse la mayor presencia relativa de mujeres en los planes de fomento del empleo. Sin embargo, las mujeres constituían al mismo tiempo el 42,0 por ciento de la población desempleada, destinataria principal de dichos planes. Es decir, el impacto diferencial sobre las paradas sería entonces mucho más débil que sobre los varones desempleados.

El alcance individual de cada uno de los programas de empleo es también muy dispar atendiendo al sexo de las personas afectadas. Se pueden distinguir claramente tres tipos de programas: a) los destinados a la contratación de jóvenes, en los que los porcentajes de mujeres oscilan entre el 39,6 por ciento en los contratos En Prácticas, el 37,9 por ciento para la Formación y el 30,4 por ciento según el R.D. 799/85; b) los destinados a la contratación de adultos, en donde la presencia femenina desciende desde el 24,8 por ciento de los contratos temporales, hasta el 10,9 por ciento en los contratos realizados a través de Convenios entre el INEM

13. Los cálculos se basan en los dato elaborados por el INEM, Estadísticas de Empleo. Un análisis pormenorizado de esta cuestión se encuentra en el estudio de J. I. Casas sobre Evaluación del impacto de las políticas de empleo sobre la situacionn laboral de la mujer, realizado para el Instituto de la Mujer. 
y los diversos Organismos y Entes Públicos; y c) los contratos a tiempo parcial, en donde la mujer es mayoritaria con un 59,7 por ciento de los contratos. Las líneas de contratación de mujeres por medio de los programas de fomento del empleo siguen, pues, las pautas de segregación clásicas para el caso de la mujer. En ese sentido, el auge de dichos programas durante el último trienio no sería ajeno al fenómeno general de precariedad del empleo, en particular el de nueva creación y que afecta directamente a la mujer.

\section{Precarización más acentuada del empleo femenino}

En efecto, los datos recientes señalan con nitidez la importancia creciente de la franja de empleos precarios entre la población activa femenina. Así, a finales de $1987,{ }^{14}$ el 23,5 por ciento de las mujeres con un empleo asalariado tenían un contrato temporal, frente al 18,2 por ciento de los varones.

Por su parte, el trabajo a tiempo parcial, que hasta principios de los años ochenta era prácticamente inexistente en nuestro país, englobaba ya al 12,1 por ciento del total de mujeres ocupadas (frente al 1,9 por ciento de los varones ocupados). La feminización del part-time sigue en España el camino ya registrado en otros países europeos, con un porcentaje del 73,6 por ciento de mujeres.

También el empleo lisa y llanamente irregular presentaba una mayor intensidad relativa entre la población femenina. Según la encuesta realizada por el Ministerio de Economía y el CIS en otoño de 1985 (SGEP y CIS, 1986), el 36 por ciento de las mujeres ocupadas se encontraban en el mercado irregular, mientras que la proporción correspondiente a los varones era del 16 por ciento. En una postetior reelaboración sobre los mismos datos, Muro et al. (1988) corregían estas cifras al tener en cuenta también la población ocupada en régimen de ayuda familiar. De este modo, las mujeres ocupadas en situación irregular serían en realidad el 42,6 por ciento, mientras que los varones serían el 19,3 por ciento.

En definitiva, el perfil prototípico de trabajador -asalariado a tiempo completo y con contrato indefinido - no puede aplicarse ni a la mitad ( 47,8 por ciento) de la población femenina ocupada, porcentaje que se eleva al 57,8 por ciento para los varones en la misma situación.

14. Datos de EPA, cuarto trimestre de 1987, principales resultados. 


\section{Elasticidad de la oferta de mano de obra femenina}

Al comienzo de este artículo aludíamos al aumento reciente de la presencia femenina en el mercado de trabajo. Este fenómeno ha estado ligado, por un lado, a la mejora de la coyuntura económica que, de esta manera, ha atraído al mercado de trabajo a buena parte del «paro desanimado» que se ha ido acumulando desde mediados de los años setenta. A este respecto, la mano de obra femenina sigue siendo el tradicional ejército de reserva, flexible y ajustable a los vaivenes de la evolución de la economía.

Pero, por otro lado, la generación de nuevos empleos durante los dos últimos años se ha producido utilizando los canales de precarización brindados por la reforma de la contratación laboral, relanzada a partir de 1984.85. Ésta parece haber sido, en lo fundamental, la vía de entrada de buena parte de las mujeres incorporadas al mercado de trabajo.

De este modo, la flexibilidad en la utilización de la mano de obra femenina no se reduce a su incorporación/expulsión del mercado de trabajo, sino también a las modalidades concretas de ocupación dentro del mismo. Las consecuencias futuras de esta forma de participación laboral de la mujer han de ser, de confirmarse las tendencias apuntadas, motivo de reflexión cuidadosa.

\section{PERSPECTIVAS DE FUTURO}

El modelo de presencia de la mujer en la esfera del trabajo parece encaminarse a reproducir algunos rasgos de la situación en otros países del norte y centro de Europa. Es decir, el incremento de la participación laboral femenina iría acompañado de una mayor precarización y marginalidad en el empleo. Se plantea entonces, dada la estructura emergente del mercado de trabajo, la disyuntiva entre una participación femenina reducida o una participación mayor, pero en condiciones laborales medias de mayor marginación. En la medida en que la estructura familiar jerarquizada no sufra alteraciones importantes, la dependencia de la mujer dentro de la misma refuerza y es a su vez reforzada por la subordinación dentro del mercado de trabajo. Junto a ello se puede asistir a una intensificación de los mecanismos de segmentación interna de la propia fuerza de trabajo femenina. Sería entonces más urgente que nunca elaborar políticas de empleo que tuvieran en cuenta esta realidad fragmentada, incidiendo no sólo en los aspectos estrictos de contratación de mano de obra, sino también de política social en otros ámbitos complementatios. 


\section{NOTES DE LECTURA}

Agüero, I., y Olano, A. (1982): «La intensa caída reciente de la fecundidad y la nupcialidad en España», en R. Conde (comp.), Familia y cambio social en España, Madrid, CIS, pp. 31-61.

Alcaide Inchausti, J. (1981): «La población española y el trabajo», Papeles de Economia Española, núm. 8, pp. 32-39.

Casas, J. 1. (1987): La participación laboral de la mujer en España, Madrid, Ministerio de Cultura, Instituto de la Mujer.

Casas, J. I. (1988): «Características del trabajo de la mujer: el caso español», Sociología del Trabajo (nueva época), núm. 3.

De Miguel, C. (1988): «La participación femenina en la actividad económica. Estructura y tendencias», Información Comercial Española, núm. 655, marzo, pp. 37-56.

Durán, M. A. (1988): «El dualismo de la economía española. Una aproximación a la economía no mercantil», Información Comercial Española, núm. 655, marzo, pp. 9-25.

Finch, J. (1983): Married to the Job. Wives' Incorporation in Men's Work, Londres, George Allen \& Unwin.

Gershuny, J. (1983), Social Innovation and the Division of Labout, Oxford, Oxford University Press.

Malo de Molina, J. L. (1983): Mercado de trabajo y estructura salarial. El caso español, 1963-1975, Madrid, IELSS.

Muro, J. et al. (1988): Análisis de las condiciones de vida y trabajo en España, Madrid, Ministerio de Economía.

Pahl, J. M. y Pahl, R. E. (1971): Managers and their Wives, Harmondsworth, Penguin.

Secretaría General de Economía y Planificación y Centro de Investigaciones Sociológicas (1986): Condiciones de vida y de trabajo en España, Madrid, CIS.

Serrano, A. y Malo de Molina, J. L. (1979): Salarios y mercado de trabajo en España, Madrid, H. Blume. 